PROCEEDINGS OF THE

AMERICAN MATHEMATICAL SOCIETY

Volume 138, Number 6, June 2010, Pages 2253-2262

S 0002-9939(10)10221-4

Article electronically published on February 12, 2010

\title{
JORDAN'S THEOREM FOR THE DIFFEOMORPHISM GROUP OF SOME MANIFOLDS
}

\author{
IGNASI MUNDET I RIERA
}

(Communicated by Richard A. Wentworth)

\begin{abstract}
Let $M$ be a compact connected $n$-dimensional smooth manifold admitting an unramified covering $\widetilde{M} \rightarrow M$ with cohomology classes $\alpha_{1}, \ldots, \alpha_{n}$ $\in H^{1}(\widetilde{M} ; \mathbb{Z})$ satisfying $\alpha_{1} \cup \cdots \cup \alpha_{n} \neq 0$. We prove that there exists some number $c$ such that: (1) any finite group of diffeomorphisms of $M$ contains an abelian subgroup of index at most $c$; $(2)$ if $\chi(M) \neq 0$, then any finite group of diffeomorphisms of $M$ has at most $c$ elements. We also give a new and short proof of Jordan's classical theorem for finite subgroups of $\operatorname{GL}(n, \mathbb{C})$, of which our result is an analogue for $\operatorname{Diff}(M)$.
\end{abstract}

\section{INTRODUCTION}

Recall the following classical theorem due to C. Jordan [J]; see also [BW, CR]:

Theorem 1.1. For any natural number $n$ there exists a constant $c$ such that any finite subgroup of $\mathrm{GL}(n, \mathbb{C})$ has an abelian subgroup of index at most $c$.

Jordan's theorem implies the next statement, proved originally by Minkowski [M]:

Corollary 1.2. For any natural $n$ there exists a constant $k$ such that any finite subgroup $\Gamma \subset \mathrm{GL}(n, \mathbb{Z})$ has at most $k$ elements.

In his beautiful survey $[\mathrm{F}$, D. Fisher asks the question, originally posed by É. Ghys, of whether Jordan's theorem is true when one replaces $\operatorname{GL}(n, \mathbb{C})$ by the diffeomorphism group of a compact smooth manifold. A related question, on which there is a good amount of literature (see $\mathrm{P}$ for a recent survey), is to study for which manifolds $M$ there is an upper bound on the size of the finite subgroups of $\operatorname{Diff}(M)$, i.e., for which $M$ Corollary 1.2 remains true if we replace $\operatorname{GL}(n, \mathbb{Z})$ by the diffeomorphism group $\operatorname{Diff}(M)$ of $M$.

Compact connected oriented surfaces provide examples of both properties, as the following (well known) theorem shows.

Theorem 1.3. Let $\Sigma$ be a compact connected oriented surface, and let $g$ be its genus.

(1) There exists a constant $c$ such that any finite subgroup of Diff $(\Sigma)$ has an abelian subgroup of index $\leq c$.

Received by the editors March 4, 2009, and, in revised form, October 12, 2009.

2010 Mathematics Subject Classification. Primary 57R50, 57S17.

(C)2010 American Mathematical Society 
(2) If $g \geq 2$, then no finite subgroup of Diff $(\Sigma)$ has more than $168(g-1)$ elements.

Proof. Let $\Gamma \subset \operatorname{Diff}(\Sigma)$ be a finite subgroup. Taking any Riemannian metric and averaging over $\Gamma$ one obtains a $\Gamma$-invariant metric on $\Sigma$. This metric defines a $\Gamma$-invariant conformal structure on $\Sigma$, which hence becomes a compact Riemann surface. Let $\operatorname{Aut}(\Sigma)$ denote the group of automorphisms of $\Sigma$ as a Riemann surface. Replacing $\Gamma$ by a subgroup of index $\leq 2$ we may assume that $\Gamma$ acts by orientationpreserving diffeomorphisms. It follows that $\Gamma \subset \operatorname{Aut}(\Sigma)$. If $g=0$, then $\operatorname{Aut}(\Sigma) \simeq$ $\operatorname{PSL}(2, \mathbb{C})$, so taking the preimage of $\Gamma$ under the projection $\operatorname{SL}(2, \mathbb{C}) \rightarrow \operatorname{PSL}(2, \mathbb{C})$ and applying Jordan's Theorem 1.1 we deduce that (1) holds in this case. If $g=1$, then any choice of base point $x \in \Sigma$ endows $\Sigma$ with a structure of an abelian group $\Sigma_{x}$, and the group of automorphisms of $\Sigma$ contains $\Sigma_{x}$ as a finite index subgroup. Hence (1) also holds if $g=1$. If $g \geq 2$, then by Hurwitz's theorem $|\operatorname{Aut}(\Sigma)| \leq 84(g-1)($ see $\S \mathrm{V} .1 .3$ in $[\mathrm{FK}])$, so $(2)$ holds in this case, and a fortiori so does (1).

In this paper we prove the following theorem.

Theorem 1.4. Let $M$ be a compact connected n-dimensional manifold admitting an unramified covering $\widetilde{M} \rightarrow M$ with cohomology classes $\alpha_{1}, \ldots, \alpha_{n} \in H^{1}(\widetilde{M} ; \mathbb{Z})$ satisfying $\alpha_{1} \cup \cdots \cup \alpha_{n} \neq 0$. There exists a constant $c$ such that:

(1) Any finite subgroup $\Gamma \subset \operatorname{Diff}(M)$ has an abelian subgroup of index $\leq c$.

(2) If $\chi(M) \neq 0$, then no finite subgroup of $\operatorname{Diff}(M)$ has more than $c$ elements.

Similar results exist in the literature; see for example DS, GLO, WW (but note that while here we consider finite groups of diffeomorphisms, the results in DS, GLO, WW] refer to finite groups of homeomorphisms). Following the terminology in DS, GLO, WW] we might call the manifolds $M$ considered in Theorem 1.4 rationally hypertoral up to a finite covering. For the more restricted class of hypertoral manifolds, statement (2) of Theorem 1.4 is contained in Corollary 2.8 of GLO.

Theorem 1.4 applies to torii $\mathbb{T}^{n}=\left(S^{1}\right)^{n}$. More generally, if $N$ is any compact connected orientable smooth manifold, then Theorem 1.4 also holds for the connected sum $N \sharp \mathbb{T}^{n}$. Indeed, if $\kappa: N \sharp \mathbb{T}^{n} \rightarrow \mathbb{T}^{n}$ is the continuous map given by contracting $N$, then $\kappa^{*} \alpha_{1} \cup \cdots \cup \kappa^{*} \alpha_{n}=\kappa^{*}\left(\alpha_{1} \cup \cdots \cup \alpha_{n}\right) \neq 0$, since $\kappa$ has degree 1 . Other examples can be obtained by taking a diffeomorphism $\phi: \mathbb{T}^{n-1} \rightarrow \mathbb{T}^{n-1}$ of finite order and constructing the mapping torus $T_{\phi}=[0,1] \times \mathbb{T}^{n-1} /\{(1, x) \sim(0, \phi x)\}$. Then $T_{\phi}$ admits a finite unramified covering by $\mathbb{T}^{n}$ (because $\phi$ has finite order), so Theorem 1.4 also applies to it.

The hypothesis that $\chi(M) \neq 0$ in (2) is certainly necessary, since for example the diffeomorphism group of $\mathbb{T}^{n}$ contains arbitrarily large finite subgroups. Statement (2) in the theorem holds for example for the connected sum of $k$ copies of $\mathbb{T}^{n}$, provided $n$ and $k$ are both $\geq 2$. So Theorem 1.4 includes as a particular case a generalization to higher dimensions of the statements in Theorem 1.3 for surfaces of genus $\geq 1$.

Theorem 1.4 implies some results on nonexistence of nontrivial actions by diffeomorphisms. In the next corollary we give two examples of such restrictions (statement (2) is not new: it is a particular case of Theorem A in [WW]). 
Corollary 1.5. Let $M$ be a compact connected $n$-dimensional manifold admitting an unramified covering $\widetilde{M} \rightarrow M$ with cohomology classes $\alpha_{1}, \ldots, \alpha_{n} \in H^{1}(\widetilde{M} ; \mathbb{Z})$ satisfying $\alpha_{1} \cup \cdots \cup \alpha_{n} \neq 0$. Let $c$ be the constant given by applying Theorem 1.4 to $M$.

(1) If $q=p^{r}>\max \{2 c, 3\}$, then any action of $\mathrm{SL}_{2}\left(\mathbb{F}_{q}\right)$ on $M$ by diffeomorphisms is trivial (here $p$ is a prime, $r \in \mathbb{N}$, and $\mathbb{F}_{q}$ is the field with $q$ elements).

(2) If $\chi(M) \neq 0$, then any action by diffeomorphisms on $M$ of a compact connected Lie group $G$ is trivial.

Proof. (1) Let $M$ satisfy the hypothesis of the corollary, and assume that there is a morphism $\phi: \mathrm{SL}_{2}\left(\mathbb{F}_{q}\right) \rightarrow \operatorname{Diff}(M)$. Let $K=\operatorname{Ker} \phi$. By Theorem 1.4 there exists an abelian subgroup $\Gamma \subset \mathrm{SL}_{2}\left(\mathbb{F}_{q}\right) / K$ of index $\leq c$. We want to prove that if $q>\max \{2 c, 3\}$, then $\phi$ is necessarily trivial. By a theorem of Jordan, $\operatorname{PSL}_{2}\left(\mathbb{F}_{q}\right)$ is simple unless $q=2,3$, which is not the case by hypothesis. So, if $\pi: \mathrm{SL}_{2}\left(\mathbb{F}_{q}\right) \rightarrow$ $\mathrm{PSL}_{2}\left(\mathbb{F}_{q}\right)$ denotes the projection map, then either $\pi(K)=\mathrm{PSL}_{2}\left(\mathbb{F}_{q}\right)$ or $K \subset \operatorname{Ker} \pi$. We claim that if $\pi(K)=\operatorname{PSL}_{2}\left(\mathbb{F}_{q}\right)$, then $K=\mathrm{SL}_{2}\left(\mathbb{F}_{q}\right)$. To see this, define

$$
A=\left(\begin{array}{cc}
1 & 0 \\
0 & -1
\end{array}\right), \quad B=\left(\begin{array}{cc}
0 & 1 \\
1 & 0
\end{array}\right) .
$$

Since $\operatorname{Ker} \pi= \pm \mathrm{Id}$, we have $\{A,-A\} \cap K \neq \emptyset$ and $\{B,-B\} \cap K \neq \emptyset$. So $K$ contains $[A, B]=-\mathrm{Id}$. Since $\operatorname{Ker} \pi= \pm \mathrm{Id} \subset K$ and $\pi(K)=\mathrm{PSL}_{2}\left(\mathbb{F}_{q}\right)$, we get $K=\mathrm{SL}_{2}\left(\mathbb{F}_{q}\right)$, as claimed. So the representation $\phi$ is trivial, and we are done. Consider now the other possibility: $K \subset \operatorname{Ker} \pi$. Let $q: \mathrm{SL}_{2}\left(\mathbb{F}_{q}\right) \rightarrow \mathrm{SL}_{2}\left(\mathbb{F}_{q}\right) / K$ be the projection. Since Ker $\pi$ is equal to the center of $\mathrm{SL}_{2}\left(\mathbb{F}_{q}\right)$ and $\Gamma$ is abelian, it follows that $\Gamma^{\prime}:=q^{-1}(K) \subset \mathrm{SL}_{2}\left(\mathbb{F}_{q}\right)$ is also abelian. Furthermore, the index of $\Gamma^{\prime}$ in $\mathrm{SL}_{2}\left(\mathbb{F}_{q}\right)$ is equal to the index of $\Gamma$ in $\mathrm{SL}_{2}\left(\mathbb{F}_{q}\right) / K$, which is $\leq c$. Let $B \subset \mathrm{SL}_{2}\left(\mathbb{F}_{q}\right)$ be the subgroup of upper triangular matrices. Then $\Gamma^{\prime \prime}:=\Gamma^{\prime} \cap B$ is an abelian subgroup of $B$. A simple argument proves that $|B| /\left|\Gamma^{\prime \prime}\right| \leq\left|\mathrm{SL}_{2}\left(\mathbb{F}_{q}\right)\right| /\left|\Gamma^{\prime}\right| \leq c$. Define $\rho: B \backslash\{ \pm \mathrm{Id}\} \rightarrow \mathbb{P}^{1}\left(\mathbb{F}_{q}\right)$ as follows:

$$
\rho\left(\begin{array}{ll}
x & y \\
0 & z
\end{array}\right)=[x-z: y] .
$$

It is easy to check that two elements in $B \backslash\{ \pm 1\}$ commute if and only if they are sent to the same element in $\mathbb{P}^{1}\left(\mathbb{F}_{q}\right)$ by $\rho$. One also checks that no fiber of $\rho$ has more than $2(q-1)$ elements. Since $B$ has $(q-1) q$ elements, it follows that no abelian subgroup of $B$ can have index $<q / 2$. Consequently $q / 2 \leq c$, and we are done.

(2) Assume that $M$ satisfies the hypothesis of the corollary and that $\chi(M) \neq 0$. Suppose that there is a nontrivial action of $S^{1}$ on $M$ by diffeomorphisms. Taking a big enough $N$ and considering the action of $e^{2 \pi \mathbf{i} / N} \in S^{1}$, one gets torsion elements in $\operatorname{Diff}(M)$ of arbitrarily high order, and this contradicts statement (2) in Theorem 1.4. Now suppose more generally that there is a nontrivial action of a compact connected Lie group $G$ on $M$. Let $\mathfrak{g}$ be the Lie algebra of $G$ and let $\psi: \mathfrak{g} \rightarrow C^{\infty}(T M)$ be the morphism induced by the infinitesimal action. Since the action of $G$ is nontrivial, $\operatorname{Ker} \psi \neq \mathfrak{g}$, so $\mathfrak{g} \backslash \operatorname{Ker} \psi$ is open and dense in $\mathfrak{g}$. Then one can take some $s \in \mathfrak{g} \backslash \operatorname{Ker} \psi$ which is tangent to a compact subgroup of $G$ isomorphic to $S^{1}$, and the restriction of the action to this subgroup will be nontrivial. This leads to a contradiction, as we have seen above. 
An interesting question, which we do not address in this paper, is to find optimal values for the constants appearing in Theorem 1.4 (for optimal estimates on the classical Jordan theorem, based on the classification of finite simple groups, see B. Weisfeiler's announcement [W], as well as A. Lubotzky's survey in [Lu]; for a study of when Hurwitz's bound used in the proof of Theorem 1.3 is optimal, see La]).

Theorem 1.4 is proved in $\$ 2$, and in $\$ 3$ we prove Jordan's Theorem 1.1 and Corollary 1.2. There already exist in the literature several proofs of Jordan's Theorem 1.1. Three reasons make us think it is worthwhile to include the present proof: it is at least as short as any other one known to the author, it is new to the best of our knowledge, and it makes the paper self-contained.

\section{Proof of Theorem 1.4}

The proof of Theorem 1.4 is divided into two parts. In Subsections 2.1 and 2.2 we prove respectively statements (1) and (2) of Theorem 1.4 in the particular case $\widetilde{M}=M$. Then in Subsection 2.3 we reduce the general statement to the case $M=\widetilde{M}$.

2.1. Proof of (1) assuming $\widetilde{M}=M$. Since there are cohomology classes $\alpha_{1}, \ldots$, $\alpha_{n} \in H^{1}(M ; \mathbb{Z})$ satisfying $\alpha_{1} \cup \cdots \cup \alpha_{n} \neq 0$, we have $H^{n}(M ; \mathbb{Z}) \neq 0$. Taking into account that $M$ is connected, this implies that $M$ is orientable. Choose an orientation of $M$. By Corollary 1.2 we can assume without loss of generality that $\Gamma$ acts trivially on $H^{1}(M ; \mathbb{Z}) /$ torsion, since the latter is isomorphic to $\mathbb{Z}^{\nu}$ for some $\nu$. Hence $\Gamma$ acts trivially on $H^{1}(M ; \mathbb{Q})$. The action of $\Gamma$ also preserves $\alpha_{1} \cup \cdots \cup \alpha_{n}$, and since the latter is a nonzero multiple of the Poincare dual of the class of a point, it follows that the action of $\Gamma$ on $M$ is by orientation-preserving diffeomorphisms. Take, for any $j$, a closed form $\widetilde{\beta}_{j} \in \Omega^{1}(M)$ representing $\alpha_{j}$ in de Rham cohomology, and define

$$
\beta_{j}=\frac{1}{|\Gamma|} \sum_{\gamma \in \Gamma} \gamma^{*} \widetilde{\beta}_{j} \in \Omega^{1}(M)
$$

Then $\beta_{j}$ is closed, it represents again $\alpha_{j}$ (since $\Gamma$ acts trivially on $H^{1}(M ; \mathbb{Q})$ ), and it is $\Gamma$-invariant. Choose a point $x_{0} \in M$ and define, for any $j$, a map $\phi_{j}: M \rightarrow S^{1}$ as:

$$
\phi_{j}(x)=\int_{\lambda_{x}} \beta_{j} \in \mathbb{R} / \mathbb{Z} \simeq S^{1},
$$

where $\lambda_{x}$ denotes any path going from $x_{0}$ to $x$ (this is well defined because $\beta_{j}$ is closed and represents an integral cohomology class). For any $\gamma \in \Gamma$ define

$$
\psi_{j}(\gamma)=\phi_{j}\left(\gamma \cdot x_{0}\right)-\phi_{j}\left(x_{0}\right) .
$$

We claim that $\psi_{j}: \Gamma \rightarrow \mathbb{R} / \mathbb{Z}$ is a morphism of groups. To see this, define for any $\gamma \in \Gamma$ the function $\gamma^{*} \phi_{j}$ as $\gamma^{*} \phi_{j}(x)=\phi_{j}(\gamma \cdot x)$. Since $\beta_{j}$ is $\Gamma$-invariant we have $d\left(\gamma^{*} \phi_{j}\right)=d \phi_{j}$, so $\gamma^{*} \phi_{j}-\phi_{j}$ is a constant. Hence we have

$$
\begin{aligned}
\phi_{j}\left(\gamma^{\prime} \gamma \cdot x_{0}\right)-\phi_{j}\left(\gamma \cdot x_{0}\right) & =\left(\left(\gamma^{\prime}\right)^{*} \phi_{j}-\phi_{j}\right)\left(\gamma \cdot x_{0}\right) \\
& =\left(\left(\gamma^{\prime}\right)^{*} \phi_{j}-\phi_{j}\right)\left(x_{0}\right) \\
& =\phi_{j}\left(\gamma^{\prime} \cdot x_{0}\right)-\phi_{j}\left(x_{0}\right) .
\end{aligned}
$$


Now we compute

$$
\begin{aligned}
\psi_{j}\left(\gamma^{\prime} \gamma\right) & =\phi_{j}\left(\gamma^{\prime} \gamma \cdot x_{0}\right)-\phi_{j}\left(x_{0}\right) \\
& =\phi_{j}\left(\gamma^{\prime} \gamma \cdot x_{0}\right)-\phi_{j}\left(\gamma \cdot x_{0}\right)+\phi_{j}\left(\gamma \cdot x_{0}\right)-\phi_{j}\left(x_{0}\right) \\
& =\phi_{j}\left(\gamma^{\prime} \cdot x_{0}\right)-\phi_{j}\left(x_{0}\right)+\phi_{j}\left(\gamma \cdot x_{0}\right)-\phi_{j}\left(x_{0}\right) \\
& =\psi_{j}\left(\gamma^{\prime}\right)+\psi_{j}(\gamma),
\end{aligned}
$$

so the claim is proved.

Recalling that $S^{1}$ has the homotopy type of the Eilenberg-MacLane space $K(\mathbb{Z}, 1)$, the map $\phi_{j}$ can be interpreted as a classifying map for $\alpha_{j}$. More precisely, orienting $S^{1}$ counterclockwise and letting $\alpha \in H^{1}\left(S^{1} ; \mathbb{Z}\right)$ be the Poincaré dual of a point, we have

$$
\phi_{j}^{*} \alpha=\alpha_{j}
$$

Define

$$
\phi=\left(\phi_{1}, \ldots, \phi_{n}\right): M \rightarrow \mathbb{T}^{n}, \quad \psi=\left(\psi_{1}, \ldots, \psi_{n}\right): \Gamma \rightarrow \mathbb{T}^{n} .
$$

Let $d$ be the nonzero integer defined by the condition that $\alpha_{1} \cup \cdots \cup \alpha_{n}=d \operatorname{PD}\left(\left[x_{0}\right]\right)$, where $\left[x_{0}\right] \in H_{0}(M ; \mathbb{Z})$ is the class represented by a point in $M$ and PD denotes the Poincaré dual. It follows from (1) that

$$
\phi_{*}[M]=d\left[\mathbb{T}^{n}\right],
$$

where $[M] \in H_{n}(M ; \mathbb{Z})$ and $\left[\mathbb{T}^{n}\right] \in H_{n}\left(\mathbb{T}^{n} ; \mathbb{Z}\right)$ are the fundamental classes in homology.

Let $\Gamma_{0}=\operatorname{Ker} \psi$.

Lemma 2.1. We have $\left|\Gamma_{0}\right| \leq d$.

Proof. It suffices to prove that $\left|\Gamma_{0}\right|$ divides $d$. It is a standard fact of differential topology (see for example $[\mathrm{BT}$, p. 47 ) that $d$ can be computed as

$$
d=\sum_{x \in \phi^{-1}(y)} \sigma(x)
$$

where $y \in \mathbb{T}^{n}$ is a regular value for $\phi$ (in particular, $\phi^{-1}(y)$ is finite). The function $\sigma$ is defined as $\sigma(x)=1$ if the differential $d \phi(x): T_{x} M \rightarrow T_{y} \mathbb{T}^{n}$ preserves the orientation and as $\sigma(x)=-1$ otherwise. Since $\phi$ is $\Gamma_{0}$-invariant, the group $\Gamma_{0}$ acts on $\phi^{-1}(y)$. We are going to prove that, for a suitable choice of regular value $y$, this action is free. Since the action of $\Gamma_{0}$ preserves the orientation, for any $x \in \phi^{-1}(y)$ and any $\gamma \in \Gamma_{0}$ we have $\sigma(\gamma \cdot x)=\sigma(x)$. This implies that $\left|\Gamma_{0}\right|$ divides $d$.

We now specify how to pick $y$. For any $\gamma \in \Gamma_{0}$ let $M^{\gamma}=\{x \in M \mid \gamma \cdot x=x\}$. We claim that $M^{\gamma}$ is a smooth submanifold of $M$. To see this, assume that $g$ is a $\Gamma$-invariant Riemannian metric on $M$. Let $\langle\gamma\rangle \subset \Gamma$ be the group generated by $\gamma$. For any $x \in M^{\gamma}$ the $g$-exponential map $\exp _{x}: T_{x} M \rightarrow M$ gives a $\langle\gamma\rangle$-equivariant diffeomorphism of a neighborhood of $0 \in T_{x} M$ with a neighborhood of $x \in M$ (since $x$ is fixed by $\gamma$, the group $\langle\gamma\rangle$ acts on $T_{x} M$ ). In particular, $\exp _{x}$ identifies a neighborhood of $x$ in $M^{\gamma}$ with a neighborhood of 0 in $\left(T_{x} M\right)^{\gamma}=\left\{v \in T_{x} M \mid \gamma \cdot v=\right.$ $v\}$. The action of $\langle\gamma\rangle$ on $T_{x} M$ is linear, so $\left(T_{x} M\right)^{\gamma} \subset T_{x} M$ is a vector subspace. Consequently, $M^{\gamma}$ is a submanifold of $M$. Since $M$ is connected, if $\gamma \neq 1$, then $M^{\gamma}$ has dimension strictly less than $\operatorname{dim} M$, so $M^{\gamma}$ has zero measure in $M$. Since $\Gamma$ is finite, $M_{f}=\bigcup_{\gamma \in \Gamma \backslash\{1\}} M^{\gamma}$ also has zero measure in $M$, and since $\phi$ is smooth and equidimensional, $\phi\left(M_{f}\right)$ has zero measure in $\mathbb{T}^{n}$. 
Let $R_{\phi} \subset \mathbb{T}^{n}$ be the set of regular values of $\phi$. By Sard's theorem (see again [BT]), $R_{\phi}$ has full measure in $\mathbb{T}^{n}$. It follows that the set $R_{\phi} \cap\left(\mathbb{T}^{n} \backslash \phi\left(M_{f}\right)\right)$ is nonempty. By construction, if $y \in R_{\phi} \cap\left(\mathbb{T}^{n} \backslash \phi\left(M_{f}\right)\right)$, then no point in $\phi^{-1}(y)$ has nontrivial stabilizer, so the action of $\Gamma_{0}$ in $\phi^{-1}(y)$ is free. This finishes the proof.

Let $\Gamma_{1}=\psi(\Gamma)$. Since $\Gamma_{1}$ is a finite subgroup of $\mathbb{T}^{n}$, it is abelian and can be generated by $\leq n$ elements. Applying the following lemma to the exact sequence of groups

$$
1 \rightarrow \Gamma_{0} \rightarrow \Gamma \rightarrow \Gamma_{1} \rightarrow 1
$$

the proof of statement (1) of the theorem is finished.

Lemma 2.2. Given $d, r \in \mathbb{N}$ there exists $c=c(d, r) \in \mathbb{N}$ with the following property. Let

$$
1 \rightarrow G_{0} \longrightarrow G \stackrel{\pi}{\longrightarrow} G_{1} \rightarrow 1
$$

be an exact sequence of finite groups, where $\left|G_{0}\right| \leq d$ and $G_{1}$ is abelian and generated by $r$ elements. Then $G$ has an abelian subgroup of index $\leq c$.

Proof. Let $j_{1}, \ldots, j_{r}$ be a set of generators of $G_{1}$. We are going to define elements $h_{1}, \ldots, h_{r} \in G$ which pairwise commute and such that, for each $i, \pi\left(h_{i}\right)=j_{i}^{k_{i}}$ for some $k_{i} \leq\left|G_{0}\right|^{i-1} \leq d^{i-1}$. Then the subgroup $G_{a} \subset G$ generated by $h_{1}, \ldots, h_{r}$ is abelian and $\pi\left(G_{a}\right) \subset G_{1}$ has index $\leq c:=d^{1+2+\cdots+(r-1)}$. Hence, $G_{a}$ has index at most $c$ in $G$.

The elements $h_{1}, \ldots, h_{r}$ are constructed recursively. First take $h_{1}$ to be any element in $\pi^{-1}\left(j_{1}\right)$. Now assume that $1<i \leq r$ and that $h_{1}, \ldots, h_{i-1} \in G$ have already been defined. Let $j$ be any element in $\pi^{-1}\left(j_{i}\right)$. Since $G_{1}$ is abelian, for any $k$ and $u$ we have $\pi\left(j^{k} h_{u} j^{-k}\right)=\pi\left(h_{u}\right)$, so by the exactness of (3) we have $j^{k} h_{u} j^{-k} h_{u}^{-1} \in G_{0}$ for each $k$. Consider the following elements in $G_{0}^{i-1}$ :

$$
\tau_{k}=\left(j^{k} h_{1} j^{-k} h_{1}^{-1}, j^{k} h_{2} j^{-k} h_{2}^{-1}, \ldots, j^{k} h_{i-1} j^{-k} h_{i-1}^{-1}\right) \in G_{0}^{i-1},
$$

where $0 \leq k \leq\left|G_{0}\right|^{i-1}$. By the pigeonhole principle, there exists $0 \leq p<$ $q \leq\left|G_{0}\right|^{i-1}$ such that $\tau_{p}=\tau_{q}$. Equating coordinates and simplifying, this implies $j^{q-p} h_{l} j^{-(q-p)}=h_{l}$ for each $l$. In other words, $h_{i}:=j^{q-p}$ commutes with $h_{1}, \ldots, h_{i-1}$. Furthermore, setting $k_{i}=q-p$ we have $\pi\left(h_{i}\right)=j_{i}^{k_{i}}$ and $k_{i} \leq\left|G_{0}\right|^{i-1}$, so all the required properties are satisfied.

2.2. Proof of (2) assuming $\widetilde{M}=M$. We proceed as in the proof of (1), but now we consider the case in which $\Gamma$ acts trivially on $H^{*}(M ; \mathbb{Z}) /$ torsion (this can be assumed, up to passing to a subgroup of index bounded above by a constant depending on $M$, again by Corollary 1.2). In this situation the group $\Gamma_{1}=\psi(\Gamma)$ has to be trivial. Indeed, any $\gamma \in \Gamma$ such that $\pi(\gamma) \neq 1$ would act on $M$ without fixed points, since it would lift an action on $\mathbb{T}^{n}$ without fixed points, and by hypothesis $\gamma$ would also act trivially on $H^{*}(M ; \mathbb{Q})$. But if $\chi(M) \neq 0$, Lefschetz's theorem implies that there does not exist any such $\gamma$. We deduce that $\Gamma=\Gamma_{0}$, so Lemma2.1 concludes the proof. 
2.3. Proof of Theorem 1.4 for arbitrary $\widetilde{M} \rightarrow M$. If $\widetilde{M}$ has more than one connected component, then there is at least one component $M^{\prime} \subset \widetilde{M}$ such that $\left.\left.\alpha_{1}\right|_{M^{\prime}} \cup \cdots \cup \alpha_{n}\right|_{M^{\prime}} \neq 0$. Hence, up to replacing $\widetilde{M}$ by $M^{\prime}$ we can assume that $\widetilde{M}$ is connected. Let $\widetilde{c}$ be the constant given by applying Theorem 1.4 to $\widetilde{M}$ (the existence of $\widetilde{c}$ is proved in Subsections 2.1 and 2.2. . Let $k$ be the degree of the covering $\widetilde{M} \rightarrow M$.

Let $\operatorname{Cov}_{k}(M)$ denote the set of isomorphism classes of (not necessarily connected) unramified coverings of $M$ of degree $k$. Picking a base point $x_{0} \in M$ and associating to each covering its monodromy, we can identify $\operatorname{Cov}_{k}(M) \simeq$ $\operatorname{Hom}\left(\pi_{1}\left(M, x_{0}\right), S_{k}\right) / S_{k}$, where $S_{k}$ is the symmetric group of $k$ letters and where $S_{k}$ acts on $\operatorname{Hom}\left(\pi_{1}\left(M, x_{0}\right), S_{k}\right)$ by conjugation. Since $M$ is compact, $\pi_{1}\left(M, x_{0}\right)$ is finitely generated, $\operatorname{so} \operatorname{Hom}\left(\pi_{1}\left(M, x_{0}\right), S_{k}\right) / S_{k}$ is finite and hence so is $\operatorname{Cov}_{k}(M)$. Let $[\widetilde{M}] \in \operatorname{Cov}_{k}(M)$ denote the equivalence class of $\widetilde{M} \rightarrow M$.

Let $\Gamma \subset \operatorname{Diff}(M)$ be a finite subgroup. Then $\Gamma$ acts on $\operatorname{Cov}_{k}(M)$ by pullback. Let $\Gamma_{0} \subset \Gamma$ be the subgroup of elements fixing $[\widetilde{M}]$. Since the orbit of $[\widetilde{M}]$ in $\operatorname{Cov}_{k}(M)$ can be identified with $\Gamma / \Gamma_{0}$, we have

$$
\sharp \Gamma / \sharp \Gamma_{0} \leq \sharp \operatorname{Cov}_{k}(M) .
$$

Let $\pi: \widetilde{M} \rightarrow M$ denote the projection, and define

$$
\Gamma_{1}=\left\{(\gamma, \phi) \in \Gamma_{0} \times \operatorname{Diff}(\widetilde{M}) \mid \pi \circ \phi=\gamma \circ \pi\right\} .
$$

Then $\Gamma_{1}$ fits in a short exact sequence

$$
1 \rightarrow \operatorname{Aut}(\widetilde{M}) \stackrel{\rho}{\longrightarrow} \Gamma_{1} \stackrel{q}{\longrightarrow} \Gamma_{0} \rightarrow 1
$$

where $\operatorname{Aut}(\widetilde{M})=\{\phi \in \operatorname{Diff}(\widetilde{M}) \mid \pi \circ \phi=\pi\}, \rho(\phi)=(1, \phi)$ and $q(\gamma, \phi)=\gamma$. The $\operatorname{group} \operatorname{Aut}(\widetilde{M})$ is finite; hence so is $\Gamma_{1}$. The map $(\gamma, \phi) \mapsto \phi$ defines an inclusion $\Gamma_{1} \hookrightarrow \operatorname{Diff}(\widetilde{M})$. Applying statement (1) of Theorem 1.4 to $\Gamma_{1}$ we deduce that there exists an abelian subgroup $\Gamma_{a b} \subset \Gamma_{1}$ of index $\leq \widetilde{c}$. Then $q\left(\Gamma_{a b}\right)$ is also abelian and has index $\leq \widetilde{c}$ as a subgroup of $\Gamma_{0}$, so, by (4), $q\left(\Gamma_{a b}\right)$ is a subgroup of $\Gamma$ of index $\leq \widetilde{c} \cdot \sharp \operatorname{Cov}_{k}(M)$. Hence statement (1) of Theorem 1.4 holds for $M$.

If $\chi(M) \neq 0$, then $\chi(\widetilde{M})=k \cdot \chi(M) \neq 0$, so we have $\sharp \Gamma_{1} \leq \widetilde{c}$. Combining (4) and (5) we deduce

$$
\sharp \Gamma \leq \sharp \Gamma_{0} \cdot \sharp \operatorname{Cov}_{k}(M) \leq \frac{\sharp \Gamma_{1} \cdot \sharp \operatorname{Cov}_{k}(M)}{\sharp \operatorname{Aut}(\widetilde{M})} \leq \frac{\widetilde{c} \cdot \sharp \operatorname{Cov}_{k}(M)}{\sharp \operatorname{Aut}(\widetilde{M})} .
$$

He have proved that the cardinal of $\Gamma$ is bounded above by a uniform constant, so statement (2) in Theorem 1.4 also holds for $M$.

\section{Proof of Jordan's Theorem 1.1 and Corollary 1.2}

3.1. Proof of Jordan's Theorem 1.1. The external structure of the proof is the same as in the usual proofs (see $[\mathrm{BW}, \mathrm{CR}]$ ). Since any finite subgroup of $\mathrm{GL}(n, \mathbb{C})$ fixes a Hermitian metric on $\mathbb{C}^{n}$, it suffices to consider finite subgroups of $\mathrm{U}(n)$. The key result is the following lemma, of which we give a new proof in Subsection 3.2 .

Lemma 3.1. There exists a neighborhood of the identity $\mathcal{U} \subset U(n)$ such that, for any finite subgroup $\Gamma \subset U(n)$, any pair of elements in $\mathcal{U} \cap \Gamma$ commute. 
We now deduce Jordan's theorem from Lemma 3.1 using a standard argument. Let $\mathcal{V} \subset \mathrm{U}(n)$ be a neighborhood of the identity such that, for any $v, v^{\prime} \in \mathcal{V}$, $v^{-1} v^{\prime} \in \mathcal{U}$. Consider Haar's measure on $\mathrm{U}(n)$, and let $c$ be the smallest integer greater than or equal to $\operatorname{Vol}(\mathrm{U}(n)) / \operatorname{Vol}(\mathcal{V})$. Let $\Gamma \subset \mathrm{U}(n)$ be a finite subgroup and let $\Gamma_{0} \subset \Gamma$ be the subgroup generated by $\mathcal{U} \cap \Gamma$. We claim that $|\Gamma| /\left|\Gamma_{0}\right| \leq c$. For otherwise there would exist elements $a_{0}, \ldots, a_{c} \in \Gamma$ such that $a_{i} a_{j}^{-1} \notin \Gamma_{0}$ for any $i \neq j$. Since $\sum_{j=0}^{c} \operatorname{Vol}\left(\mathcal{V} \cdot a_{j}\right)>\operatorname{Vol}(\mathrm{U}(n))$, there must exist $i \neq j$ such that $\mathcal{V} \cdot a_{i} \cap \mathcal{V} \cdot a_{j} \neq \emptyset$. So $v a_{i}=v^{\prime} a_{j}$ for some $v, v^{\prime} \in \mathcal{V}$, which implies $\Gamma \ni a_{i} a_{j}^{-1}=$ $v^{-1} v^{\prime} \in \mathcal{U}$, a contradiction.

3.2. Proof of Lemma 3.1. Let $\mathcal{S}=\left\{\left(e_{i}\right)\right\}$ be the Seifert variety of unitary basis of $\mathbb{C}^{n}$. Assigning to each vector its coordinates in the canonical basis we get an embedding $\mathcal{S} \subset \mathbb{C}^{n^{2}}$. Consider the restriction to $\mathcal{S}$ of the Riemannian metric on $\mathbb{C}^{n^{2}}$. The group $\mathbb{T}=\left(S^{1}\right)^{n}$ acts freely and isometrically on $\mathcal{S}$ by componentwise multiplication, $\left(\theta_{i}\right) \cdot\left(e_{i}\right)=\left(\theta_{i} e_{i}\right)$. Let $\mathcal{P}$ be the manifold $\mathcal{S} / \mathbb{T}$, endowed with the quotient Riemannian metric. Let $d$ denote the distance function between points in $\mathcal{P}$. The diagonal action of $\mathrm{U}(n)$ on $\mathcal{S}$ given by $A\left(e_{i}\right)=\left(A e_{i}\right)$ descends to an isometric action on $\mathcal{P}$. For any $x \in \mathcal{P}$ and $r>0$ we denote by $B(x, r) \subset \mathcal{P}$ the ball of radius $r$ centered at $x$.

We say that two elements $\left[e_{i}\right],\left[f_{i}\right] \in \mathcal{P}$ are partially orthogonal if there exists a nonempty set $I \subset\{1, \ldots, n\}$ such that the subspaces $V_{I}=\left\langle e_{i}\right\rangle_{i \in I}, W_{I}=\left\langle f_{i}\right\rangle_{i \in I} \subset$ $\mathbb{C}^{n}$ satisfy $V_{I}^{\perp} \cap W_{I} \neq 0$, where the superscript ${ }^{\perp}$ denotes the Hermitian orthogonal. Let

$$
\delta_{0}=\inf \{d(e, f) \mid e, f \in \mathcal{P} \text { are partially orthogonal }\} .
$$

Lemma 3.2. There exists $k \in \mathbb{N}$ such that for any $\delta>0$, any $x \in \mathcal{P}$, and any set $W \subset B(x, \delta)$ with $>k$ elements, we have $d\left(w, w^{\prime}\right)<\min \left\{\delta, \delta_{0}\right\}$ for some distinct $w, w^{\prime} \in W$.

Proof. Since $\mathcal{P}$ is compact, there exist $r_{0}, C, C^{\prime}>0$ such that for any $0 \leq r \leq r_{0}$ and any $x \in \mathcal{P}$ we have $C r^{\nu} \leq \operatorname{Vol}(B(x, r)) \leq C^{\prime} r^{\nu}$, where $\nu=\operatorname{dim} \mathcal{P}$. If $k \geq$ $k_{0}=3^{\nu} C^{\prime} C^{-1}$ and $r \leq 2 r_{0} / 3$, then for any $y_{0}, \ldots, y_{k} \in B(x, r)$ there exist $i \neq j$ such that $d\left(y_{i}, y_{j}\right)<r$ : otherwise the balls $B\left(y_{1}, r / 2\right), \ldots, B\left(y_{k}, r / 2\right)$ would be disjoint and contained in $B(x, 3 r / 2)$, contradicting the volume estimate. If $k \geq$ $k_{1}=\operatorname{Vol}(\mathcal{P}) C^{-1}\left(\min \left\{\rho_{0}, 2 r_{0} / 3\right\} / 2\right)^{-\nu}$, then in any set $\left\{z_{0}, \ldots, z_{k}\right\} \subset \mathcal{P}$ there is at least a pair of points at distance $<\min \left\{\rho_{0}, 2 r_{0} / 3\right\}$. Hence, taking $k \geq \max \left\{k_{0}, k_{1}\right\}$ we find that the statement of the lemma holds true.

Let $k$ be as in the previous lemma and let $\epsilon>0$ be small enough so that the restriction of the map $z \mapsto z^{k}$ to the open disk $D=\{z \in \mathbb{C}|| z-1 \mid<\epsilon\}$ is injective. Define

$$
\mathcal{U}=\{A \in \mathrm{U}(n) \mid \operatorname{det}(A-\lambda \mathrm{Id})=0 \Rightarrow \lambda \in D\} .
$$

If $B \in \mathcal{U}, 1 \leq r \leq k$, and $v \in \mathbb{C}^{n}$, then $v$ is an eigenvector of $B$ if and only if it is an eigenvector of $B^{r}$. Since two matrices commute iff there exists a basis in which both diagonalize, this implies:

Lemma 3.3. If $A, B \in \mathcal{U}$ and $1 \leq r \leq k$, then $A B=B A$ if and only if $A B^{r}=$ $B^{r} A$. 
We now prove that the set $\mathcal{U}$ has the property stated in Lemma 3.1 Let $\Gamma \subset \mathrm{U}(n)$ be a finite subgroup, and assume that some elements $X, Y \in \Gamma \cap \mathcal{U}$ do not commute. By Lemma 3.3. $(X, Y)$ belongs to the set

$$
\mathcal{N}=\{(X, Y) \in(\Gamma \cap \mathcal{U}) \times(\Gamma \cap \mathcal{U}) \mid X Y \neq Y X\},
$$

which consequently is nonempty. $\mathcal{N}$ is also finite, because $\Gamma$ is finite.

For any $X \in \mathrm{U}(n)$ let $\mathcal{E}(X)$ be the set of $e \in \mathcal{P}$ represented by a unitary basis consisting of eigenvectors of $X$. Given $X, Y \in \mathrm{U}(n)$ we define

$$
d_{\sigma}(X, Y)=\inf \{d(e, f) \mid e \in \mathcal{E}(X), f \in \mathcal{E}(Y)\} .
$$

Clearly, $X$ and $Y$ commute iff $d_{\sigma}(X, Y)=0$. Let $\delta=\min \left\{d_{\sigma}(X, Y) \mid(X, Y) \in \mathcal{N}\right\}$. Since $\mathcal{N}$ is finite, $\delta>0$, and there exists some $(A, B) \in \mathcal{N}$ satisfying $d_{\sigma}(A, B)=$ $\delta$. Since $\mathcal{E}(A), \mathcal{E}(B)$ are compact, there exist $e \in \mathcal{E}(A)$ and $f \in \mathcal{E}(B)$ satisfying $d(e, f)=\delta$. Then the elements $e, B e, \ldots, B^{k} e \in \mathcal{P}$ are pairwise distinct: if $B^{p} e=$ $B^{q} e$ for some $p \neq q$, then $e=B^{q-p} e$, so $B^{q-p}$ commutes with $A$, and by Lemma 3.3 this would contradict the assumption that $(A, B) \in \mathcal{N}$. Since $B f=f$ and $B$ acts on $\mathcal{P}$ by isometries, we have $d\left(B^{j} e, f\right)=\delta$ for each $j$, so by the choice of $k$ there exists some $p \neq q$ such that $d\left(B^{p} e, B^{q} e\right)<\min \left\{\delta, \delta_{0}\right\}$. Let $l=q-p$. We have $B^{l} e \in \mathcal{E}\left(B^{l} A B^{-l}\right)$, so

$$
d_{\sigma}\left(A, B^{l} A B^{-l}\right) \leq d\left(e, B^{l} e\right)<\delta .
$$

We claim that $\left(A, B^{l} A B^{-l}\right) \in \mathcal{N}$. Combining this claim with (6) we obtain a contradiction with the choice of $\delta$, thus finishing the proof of the lemma. To prove the claim, suppose that $A$ and $B^{l} A B^{-l}$ commute. Let $\left\{\lambda_{j}\right\}$ be the eigenvalues of $A$ and let $V_{j}=\operatorname{Ker}\left(A-\lambda_{j}\right)$. Since $d\left(e, B^{l} e\right)<\delta_{0}, e$ and $B^{l} e$ are not partially orthogonal so, for any $j, B^{l}\left(V_{j}\right)$ is the graph of a map $f: V_{j} \rightarrow V_{j}^{\perp}$. Since $A$ commutes with $B^{l} A B^{-l}$, for any $r$ such that $f\left(V_{j}\right) \nsubseteq V_{r}^{\perp}$ we have $\lambda_{r}=\lambda_{j}$; hence $r=j$, so $f=0$. It follows that $B^{l}$ commutes with $A$, contradicting (by Lemma 3.3) the fact that $(A, B) \in \mathcal{N}$.

3.3. Proof of Corollary 1.2. Let $\Gamma \subset \mathrm{GL}(n ; \mathbb{Z})$. By Theorem 1.1 there exists an abelian subgroup $\Gamma_{0} \subset \Gamma$ of index $\leq c$. Since $\Gamma_{0}$ is abelian there exists a basis $e$ of $\mathbb{C}^{n}$ in which all elements of $\Gamma_{0}$ are diagonal matrices, so it suffices to prove that the set of possible eigenvalues of elements in $\Gamma_{0}$ is finite. The eigenvalues of any $\gamma \in \Gamma_{0}$ are roots of unity (because $\Gamma_{0}$ is finite) and its characteristic polynomial has integer coefficients, so its eigenvalues belong to the set $\Lambda_{n} \subset \mathbb{C}$ of roots of unity $\rho$ for which there exists a monic polynomial $P \in \mathbb{Z}[x]$ of degree $\leq n$ satisfying $P(\rho)=0$. Assume that $\rho \in \Lambda_{n}$ and that $P \in \mathbb{Z}[x]$ has degree $\leq \bar{n}$ and satisfies $P(\rho)=0$. Up to replacing $P$ by the greatest common divisor of $P$ and $x^{n}-1$, we can assume that all roots of $P$ have modulus 1 . So, if $k$ denotes the degree of

$P$, the coefficient of $x^{j}$ in $P$ has modulus $\leq\left(\begin{array}{l}k \\ j\end{array}\right)$. Since the coefficients of $P$ are integers, it follows that there are finitely many polynomials $P \in \mathbb{Z}[x]$ of degree $k$ which can arise in this way. Consequently, $\Lambda_{n}$ is finite.

\section{ACKNOWLEDGMENTS}

I wish to thank Jaume Amorós for useful conversations and the TIFR (Mumbai, India), where this paper was written, for its hospitality and excellent working conditions. 


\section{REFERENCES}

[BT] R. Bott, L.W. Tu, Differential Forms in Algebraic Topology, Graduate Texts in Mathematics, 82, Springer, 1982. MR658304 (83i:57016)

[BW] W.M. Boothby, H.-C. Wang, On the finite subgroups of connected Lie groups, Comment. Math. Helv. 39 (1965), 281-294. MR0180622(31:4856)

[CR] C.W. Curtis, I. Reiner, Representation Theory of Finite Groups and Associative Algebras, reprint of the 1962 original, AMS Chelsea Publishing, Providence, RI, 2006. MR2215618 (2006m:16001)

[DS] H. Donnelly, R. Schultz, Compact group actions and maps into aspherical manifolds, Topology 21 (1982), no. 4, 443-455. MR670746 (84k:57024)

[FK] H.M. Farkas, I. Kra, Riemann Surfaces, Graduate Texts in Mathematics, 71, SpringerVerlag, New York-Berlin, 1980. MR583745 (82c:30067)

[F] D. Fisher, Groups acting on manifolds: Around the Zimmer program, preprint, arXiv:0809.4849, to appear in Festschrift for R.J. Zimmer.

[GLO] D. Gottlieb, K.B. Lee, M. Özaydin, Compact group actions and maps into $K(\pi, 1)$-spaces, Trans. Amer. Math. Soc. 287 (1985), no. 1, 419-429. MR766228(86h:57034)

[J] C. Jordan, Mémoire sur les équations différentielles linéaires à intégrale algébrique, J. Reine Angew. Math. 84 (1878), 89-215.

[La] M. Larsen, How often is 84( $g-1)$ achieved?, Israel J. Math. 126 (2001), 1-16. MR.1882031 (2002m:30056)

[Lu] A. Lubotzky, The Mathematics of Boris Weisfeiler, Notices Amer. Math. Soc. 51 (2004), no. 1, 31-32. MR2022673

[M] H. Minkowski, Zur Theorie der positiven quadratischen Formen, Crelle Journal für die reine und angewandte Mathematik, 101 (1887), 196-202. (See also Collected Works. I, 212-218, Chelsea Publishing Company, 1967.)

[P] V. Puppe, Do manifolds have little symmetry?, J. Fixed Point Theory Appl. 2 (2007), no. 1, 85-96. MR2336501 (2009b:57065)

[WW] R. Washiyama, T. Watabe, On the degree of symmetry of a certain manifold, J. Math. Soc. Japan 35 (1983), no. 1, 53-58. MR679074 (85d:57034)

[W] B. Weisfeiler, Post-classification version of Jordan's theorem of finite linear groups, Proc. Nat. Acad. Sci. USA 81 (1984), no. 16, Phys. Sci., 5278-5279. MR758425 (85j:20041)

Departament d’Àlgebra i Geometria, Facultat de Matemàtiques, Universitat de Barcelona, Gran Via de les Corts Catalanes 585, 08007 Barcelona, Spain

E-mail address: ignasi.mundet@ub.edu 